\title{
Reports
}

\section{Macular Thickness as a Potential Biomarker of Mild Alzheimer's Disease}

Although several postmortem findings in the retina of patients with Alzheimer's disease (AD) are available, ${ }^{1}$ new biomarkers for early diagnosis and follow-up of AD are still lacking. It has been postulated that the defects in the retinal nerve fiber layer (RNFL) may be the earliest sign of $\mathrm{AD}$, even before damage to the hippocampal region that affects memory. ${ }^{2}$ This fact may reflect retinal neuronal-ganglion cell death and axonal loss in the optic nerve in addition to aging.

Changes in longitudinal optical coherence tomography (OCT) measurements of RNFL can act as a surrogate marker of axonal health, making OCT an invaluable tool for measuring axonal loss as a biomarker. ${ }^{2}$ The goal of this study was to examine the thickness of the macular and peripapillary RNFL with OCT to determine the most predictive area affected in patients with mild AD, Geriatric Depression Scale-4, Reisberg scale, and make comparisons using normal subjects.

Twenty patients with mild AD and 28 age-matched control subjects from the Geriatric Unit in the Hospital Clínico San Carlos, Madrid, Spain, were studied. The AD patients met the criteria for $\mathrm{AD}$ according to the National Institute of Neurological and Communicative Disorders and Stroke-Alzheimer's Disease and Related Disorders Association and the Diagnostic and Statistical Manual of Mental Disorders IV, having mild cognitive impairment according to the Clinical Dementia Rating scale. Informed consent was obtained from both groups. The research followed the tenets of the Declaration of Helsinki, and the protocol was approved by the local ethics committee.

The inclusion criteria for patients were: being free of ocular disease and systemic disorders affecting vision; best-corrected visual acuity (BCVA) of 20/40; \pm 5 spherocylindrical refractive error; and intraocular pressure of $<20 \mathrm{mmHg}$. All the subjects underwent a complete ophthalmologic examination, including visual acuity, refraction, anterior and posterior segment biomicroscopy, intraocular pressure measurement, dilated fundus examination, and OCT. The RNFL thickness and macular thickness were measured by OCT Model 3D OCT-1000 (Topcon, Japan) after pupil dilatation. The analysis area was centered manually and the absence of segmentation errors was confirmed for each scan. One eye of each patient assigned at random was analyzed. In the peripapillary area the average thickness $\left(360^{\circ}\right)$ and the temporal $\left(316^{\circ}-45^{\circ}\right)$, superior $\left(46^{\circ}-135^{\circ}\right)$, nasal $\left(136^{\circ}-225^{\circ}\right)$, and inferior $\left(226^{\circ}-315^{\circ}\right)$ quadrant thicknesses were evaluated. Macular RNFL thickness data were displayed in 3 concentric rings centered in the foveola, distributed as follows: A central macular ring, $1 \mathrm{~mm}$ from the fovea; an inner macular ring, 3 $\mathrm{mm}$ from the fovea; and an outer macular ring, $6 \mathrm{~mm}$ from the fovea The inner and outer rings were each divided into 4 quadrants (superior, inferior, nasal, and temporal (Fig 1).

The data are reported as mean values \pm standard deviations. The differences between groups were analyzed using the MannWhitney $U$ test. Sensitivity at $90 \%$ specificity and receiver operating characteristics (ROCs) analysis for discriminating between healthy and mild AD patients were calculated for the RNFL thickness in all the areas studied. Data were processed in a SPSS 19.0. $P<0.05$ was considered statistically significant.
There were no statistically significant differences in age or gender between the groups. The Mini-Mental State Examination (MMSE) scores in mild AD patients significantly decreased in comparison to control subjects. All the patients had MMSE values of $>17$ (Table 1 ; available at www.aaojournal.org).

OCT. Compared with controls, in patients with mild AD: (1) the thickness values for the central ring and the 4 inner quadrants were significantly decreased $(P<0.05)$; (2) the RNFL thickness of the outer macular quadrants were diminished; (3) only the values of the outer temporal quadrant were significantly lower $(P<0.05)$; (4) the total macular volume was significantly reduced $(P<0.05)$; (5) peripapillary RNFL-thickness values and signal strength did not differ between groups $(P>0.05$; Fig 2A-D; Table 2; available at www.aaojournal.org).

ROCs Curves. A significant relationship was found between the central and inner macular RNFL thickness in all areas. The analysis of the ROCs curves showed that, for AD, the RNFL thickness in the central and inner macular quadrants had the widest areas under the ROCs curves for all the parameters analyzed $(0.743-0.771 ; P<0.05)$. The inner superior quadrant had the strongest correlation $(r=0.821 ; P<0.01)$ followed by inner temporal quadrant $(r=0.806 ; P<0.01)$. With respect to the total macular area, the analysis revealed a strong correlation $(r=0.742$; $P<0.01$; Fig 2E-H; available at www.aaojournal.org).

Widespread axonal degeneration in the optic nerve had been found both in postmortem and in vivo studies of $\mathrm{AD}$ patients. ${ }^{1-3}$ In the present study, our mild AD patients, compared with a control group, had a statistically significant decrease in RNLF thickness, of some macular regions and in the total macular volume. These data are consistent with histopathologic studies ${ }^{1,3}$ demonstrating a decrease of $25 \%$ of neurons in the ganglion-cell layer of the macula; the greatest decrease being detected in the temporal region of the fovea $(52 \%)$.

The first reports on retinal involvement in AD stated that retinal alterations occur as a consequence of a retrograde pathway. However, it has recently been reported that neuroinflammation induced by beta-amyloid-, APP- and tau deposits in the RGC- and IPL layers could be causing direct retinal degeneration that may account, at least in part, for the OCT alterations reported here. On the other hand, the macular region contains the highest RGC concentration of the retina, among which parvocellular RGC is the predominant cell type. Notably, psychophysical exploration of the parvocellular pathway are affected in AD. ${ }^{4,5}$

Our data showed values for the macular RNFL thickness and total macular volume measured by OCT to have highly significant sensitivity and specificity for differentiating mild AD patients from healthy subjects. The most sensitive area was the superior inner macula, followed by the temporal inner macula, with ROCs values of $85 \%$ and $80 \%$, respectively. Thus, according to our data, the superior inner macular RNFL thickness seems to have the highest diagnostic value in $\mathrm{AD}$ neurodegeneration. To our knowledge, this is the first available report on the area of the central retina that has a higher predictive ability in mild AD patients. Further studies to confirm this preliminary data are needed.

In comparison with other studies on histopathology and on RNFL analysis by OCT in AD, our patients had: (1) similar mean 


\section{Ophthalmology Volume a, Number a, Month 2014}

A

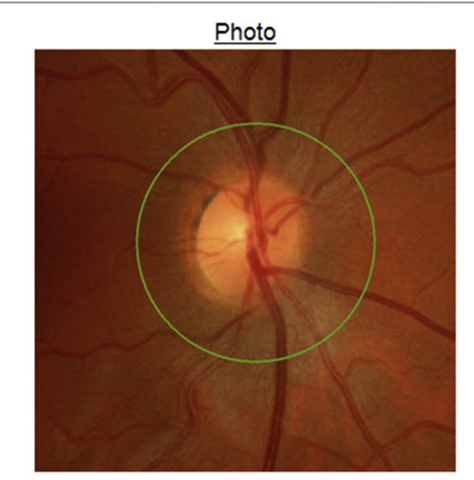

PERIPAPILLARY OCT
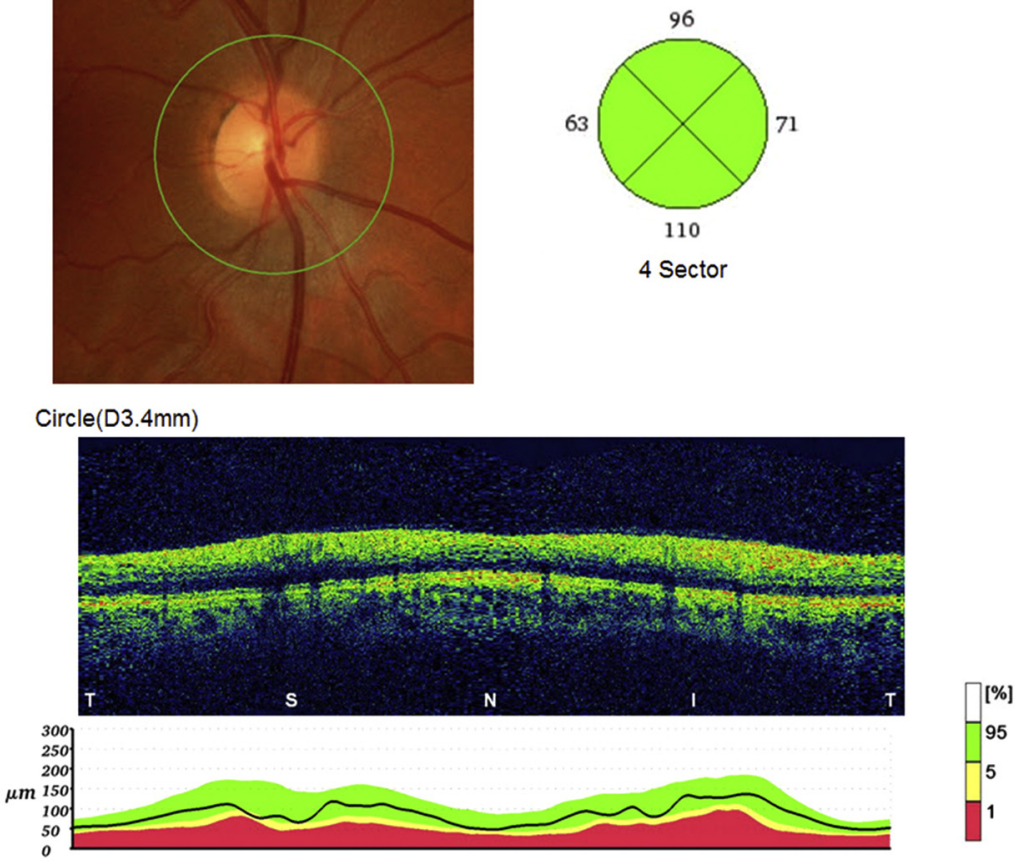

B

MACULAR OCT
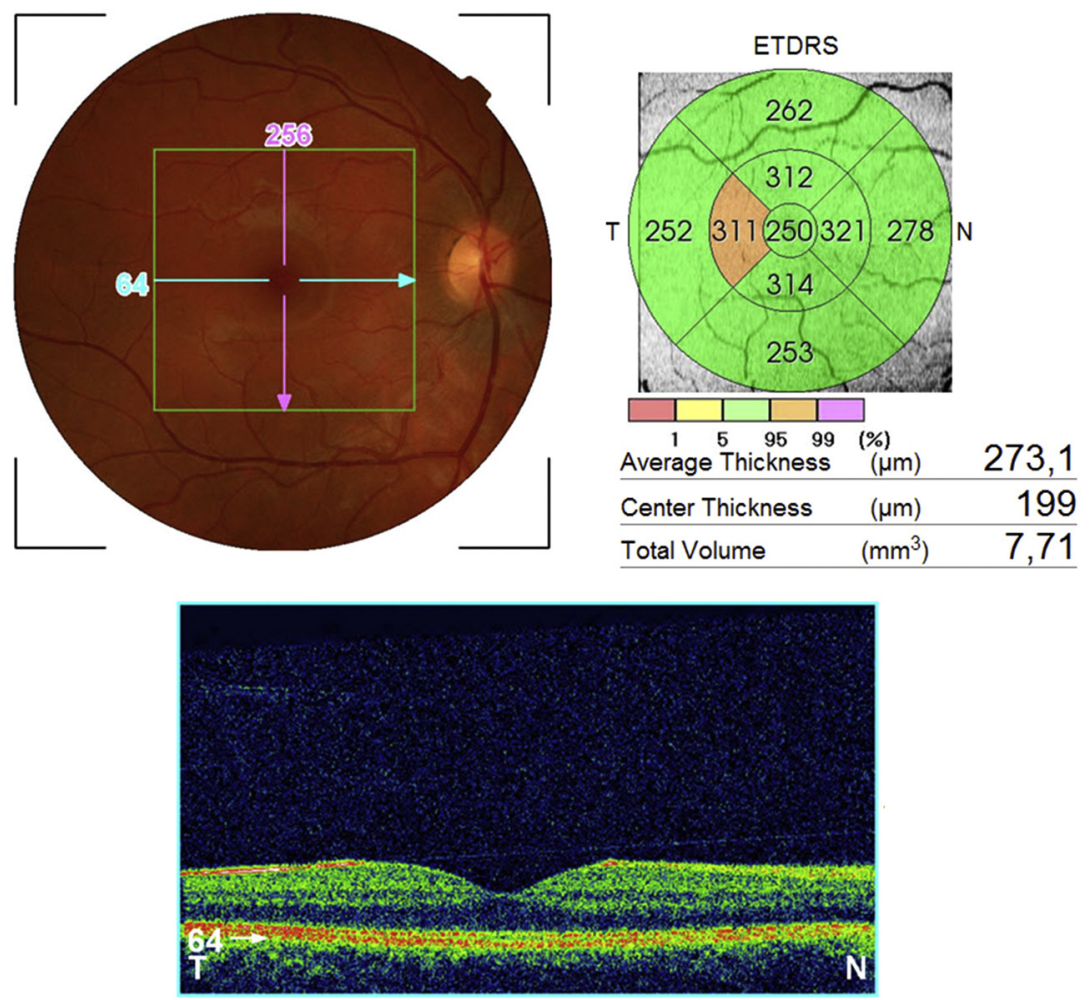

Figure 1. Retinal nerve fiber layer thickness analysis (RNFL). Optical coherence tomography (OCT) study. (A) Peripapillary OCT. Upper left: Peripapillary retinography with a green circle marking the retinal tissue considered for analysis. Upper right: diagram of the peripapillary quadrants analyzed: temporal quadrant $\left(316^{\circ}\right.$ to $\left.45^{\circ}\right)$, superior quadrant $\left(46^{\circ}\right.$ to $\left.135^{\circ}\right)$, nasal quadrant $\left(136^{\circ}\right.$ to $\left.225^{\circ}\right)$, inferior quadrant $\left(226^{\circ}\right.$ to $\left.315^{\circ}\right)$. Bottom: retinal b-scan and diagram of thickness normality. (B) Macular OCT. Upper left. Central retinography with a green square marking the retinal tissue considered for analysis. Upper right: diagram showing the concentric rings and quadrants considered for analysis of the macular RNFL thickness and measurements automatically provided by the analyser. Bottom: retinal b-scan of the macula. ETDRS = Early Treatment Diabetic Retinopathy Study. 


\section{Reports}

age values, so differences in OCT findings seems not to be attributable to aging and, (2) higher mean MMSE values seem to indicate that they are taking place in early stages of AD.

Based on our data, we propose that the first affected area of the retina in mild AD may be the macular area. As the neurodegeneration progresses, a significant decline in peripapillary RNFL thickness becomes apparent. However, whether or not the macula is really the first area involved in early $\mathrm{AD}$, or simply the first place with enough RGCs to discern an effect deserves further investigation.

We suggest that with OCT we can detect consistent macular changes that can be of significant value for evaluating $\mathrm{AD}$ patients. The retina, being part of the central nervous system and offering easy accessibility, encourages its use as a potential biomarker for $\mathrm{AD}$ diagnosis and progression.

\author{
Elena S. Garcia-Martin, MSC \\ Blanca Rojas, MD, PhD ${ }^{1,2}$ \\ ANA I. RAMIREZ, PHD ${ }^{1,3}$ \\ Rosa de Hoz, MD, PhD ${ }^{1,3}$ \\ JuAN J. SAlazAr, PhD ${ }^{1,3}$ \\ Raquel Yubero, PhD ${ }^{4}$ \\ Pedro GIL, MD, PhD ${ }^{2,4}$ \\ Alberto Triviño, MD, PhD ${ }^{1,2}$ \\ Jose M. RAmirez, MD, PhD ${ }^{1,2}$
}

\begin{abstract}
${ }^{1}$ Instituto de Investigaciones Oftalmológicas Ramón Castroviejo, Universidad Complutense de Madrid, Madrid, Spain; ${ }^{2}$ Facultad de Medicina, Universidad Complutense de Madrid, Madrid, Spain; ${ }^{3}$ Facultad de Óptica y Optometría, Universidad Complutense de Madrid, Madrid, Spain; ${ }^{4}$ Hospital Clínico San Carlos, Madrid, Spain Financial Support: Redes temáticas de investigación cooperativa en salud (RETICs) Prevención, detección precoz y tratamiento de la patología ocular prevalente degenerativa y crónica (grant ISCIII RD12/ 0034/0002, Spanish Ministry of Science and Innovation). The author(s) have no proprietary or commercial interest in any materials discussed in this article.
\end{abstract}

\section{References}

1. Hinton DR, Sadun AA, Blanks JC, et al. Optic-nerve degeneration in Alzheimer's disease. N Engl J Med 1986;315:485-7.

2. He XF, Liu YT, Peng C, et al. Optical coherence tomography assessed retinal nerve fiber layer thickness in patients with Alzheimer's disease: a meta-analysis. Int J Ophthalmol 2012;5:401-5.

3. Blanks JC, Torigoe Y, Hinton DR, et al. Retinal pathology in Alzheimer's disease. I. ganglion cell loss in foveal/parafoveal retina. Neurobiol Aging 1996;17:377-84.

4. Tzekov RT, Mullan M. Vision function abnormalities in alzheimer's disease. Surv Ophthalmol 2013; In press: http://dx.doi. org/10.1016/j.survophthal.2013.10.002.

5. Koronyo Y, Salumbides BC, Black KL, et al. Alzheimer's disease in the retina: Imaging retinal a $\beta$ plaques for early diagnosis and therapy assessment. Neurodeg Dis 2012;10:285-93. 
Ophthalmology Volume a, Number a, Month 2014

Table 1. Demographic and Clinical Data of the Study Groups

\begin{tabular}{lccc}
\hline \multicolumn{1}{c}{ Variable } & AD $(\mathbf{n}=20)$ & Control $(\mathbf{n}=28)$ & $P$ \\
\hline Age (y), mean \pm SD & $79.3 \pm 4.1$ & $72.1 \pm 5.1$ & 0.274 \\
Gender & & & 0.874 \\
$\quad$ Male & 12 & 9 & \\
$\quad$ Female & Caucasian & Caucasian & \\
Race & $23.7 \pm 3.3$ & $28.1 \pm 2.1$ & $0.009 *$ \\
MMSE (mean \pm SD) & & & \\
& & & \\
AD $=$ Alzheimer's disease; MMSE $=$ Mini-Mental State Examination; \\
$\mathrm{SD}=$ standard deviation. \\
*P $P .01$.
\end{tabular}




\section{Reports}

A

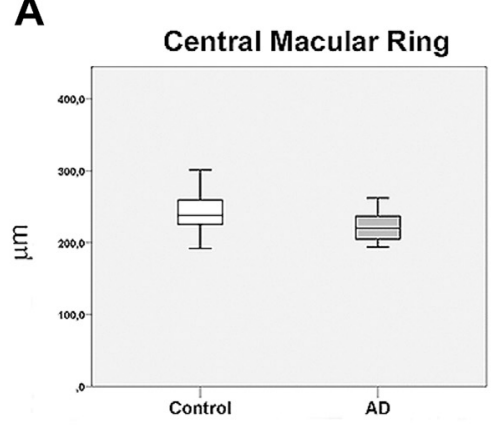

C

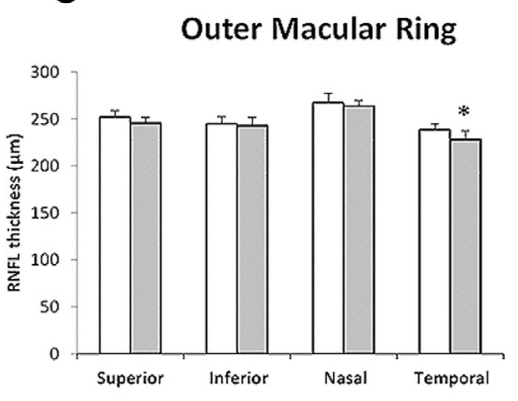

E

Central macular ring / macular volume

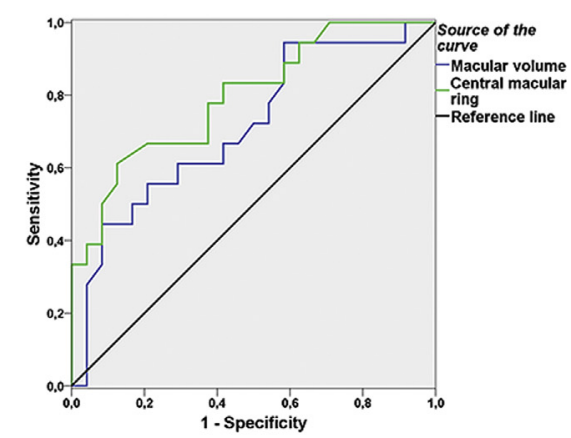

G

Outer macular quadrants

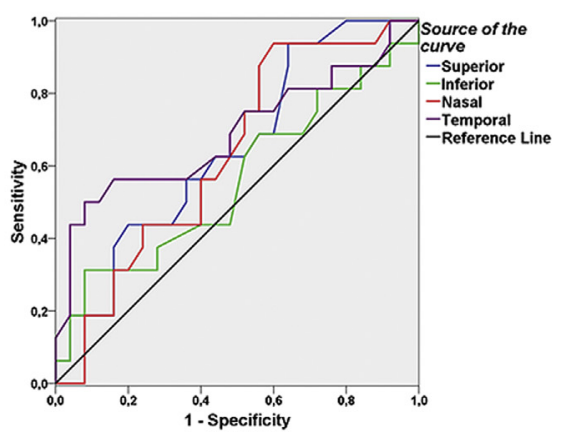

B

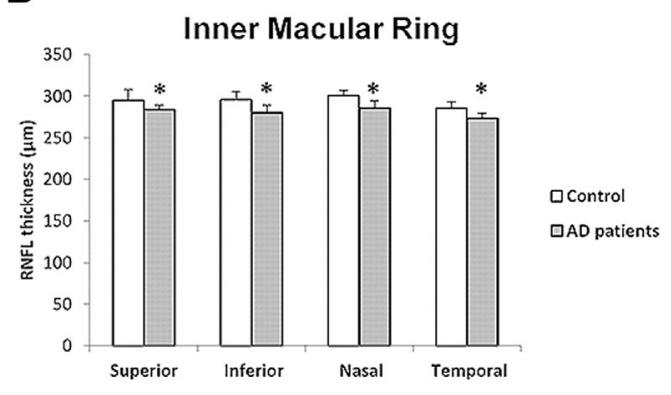

D

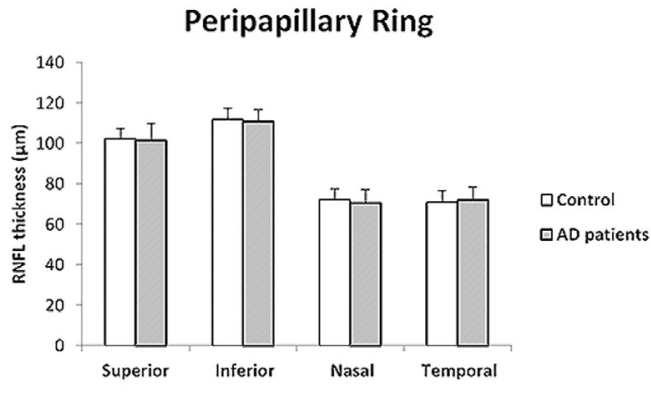

$\mathbf{F}$

Inner macular quadrants

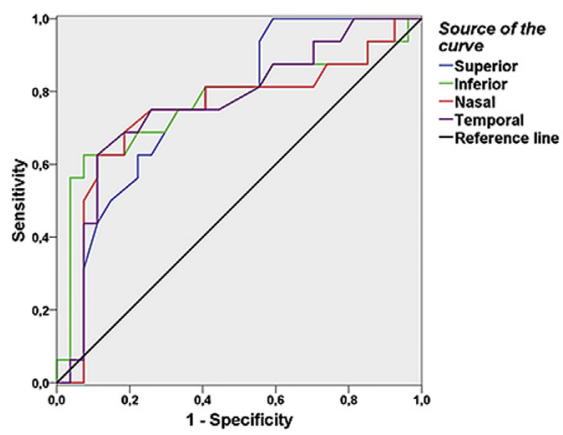

$\mathbf{H}_{\text {Peripapillary quadrants }}$

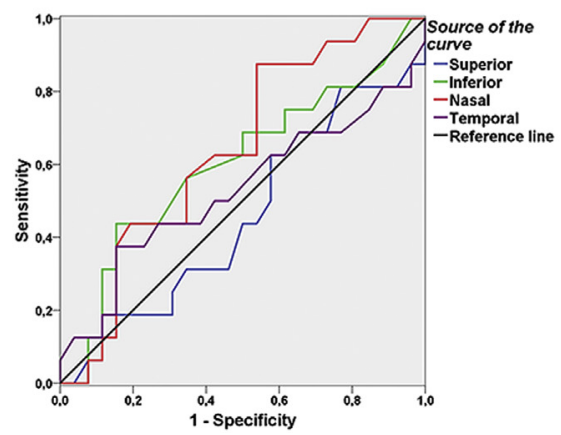

Figure 2. Mean data of retinal nerve fiber layer (RNFL) thickness against eye quadrants assessed with optical coherence tomography and areas under the receiver operating characteristics (ROCs) curve. (A-D) Central macular ring (1 mm from the fovea). Horizontal lines, bottom to top: 10th, 25th, 50th (median), 75th, and 90th percentiles (A). Inner macular ring (3 mm from the fovea) (B). Outer macular ring (6 mm from the fovea) (C). Peripapillary retinal nerve-fiber layer (D). (E-F) Areas under the ROCs curves in discriminating between mild Alzheimer disease patients and control subjects, from the central macular ring and total macular volume $(\mathrm{E})$, inner macular quadrants $(\mathrm{F})$, outer macular quadrants $(\mathrm{G})$ and peripapillary quadrants $(\mathrm{H})$. $* P<0.01$. 


\section{Ophthalmology Volume a, Number $\mathbf{n}$, Month 2014}

Table 2. RNFL Thickness and Total Macular Volume

\begin{tabular}{|c|c|c|c|c|}
\hline Retinal area of study & $\mathrm{AD}$ group (mean $\pm \mathrm{SD})$ & Control group (mean \pm SD) & $\%$ RNFL decrease & $P$ \\
\hline Foveal thickness $(\mu \mathrm{m})$ & $221.2 \pm 21.6$ & $243.7 \pm 24.8$ & 9.24 & $0.015 *$ \\
\hline \multicolumn{5}{|l|}{ Inner macular quadrant $(\mu \mathrm{m})$} \\
\hline Superior area & $283.4 \pm 11.1$ & $294.9 \pm 18.1$ & 3.91 & $0.002 *$ \\
\hline Inferior area & $279.8 \pm 18.1$ & $295.8 \pm 13.5$ & 5.40 & $0.002 *$ \\
\hline Nasal area & $285.6 \pm 17.2$ & $300.1 \pm 15.1$ & 4.83 & $0.007 *$ \\
\hline Temporal area & $273.1 \pm 12.7$ & $285.2 \pm 14.6$ & 4.22 & $0.002 *$ \\
\hline \multicolumn{5}{|l|}{ Outer macular quadrant $(\mu \mathrm{m})$} \\
\hline Superior area & $245.4 \pm 12.5$ & $252.1 \pm 13.7$ & 2.65 & 0.084 \\
\hline Inferior area & $242.8 \pm 17.4$ & $245.2 \pm 13.9$ & 0.99 & 0.531 \\
\hline Nasal area & $263.7 \pm 12.1$ & $267.5 \pm 19.1$ & 1.41 & 0.110 \\
\hline Temporal area & $228.0 \pm 18.8$ & $238.5 \pm 12.3$ & 4.43 & $0.009 *$ \\
\hline \multicolumn{5}{|l|}{ Peripapillary thickness $(\mu \mathrm{m})$} \\
\hline Superior area & $101.4 \pm 16.5$ & $102.2 \pm 10.8$ & 0.77 & 0.404 \\
\hline Inferior area & $110.8 \pm 11.1$ & $111.8 \pm 10.8$ & 0.83 & 0.353 \\
\hline Nasal area & $70.5 \pm 12.8$ & $72.3 \pm 10.6$ & 2.01 & 0.283 \\
\hline Temporal area & $71.8 \pm 12.5$ & $70.6 \pm 11.6$ & -1.67 & 0.616 \\
\hline Total macular volume (cubic mm) & $7.1 \pm 0.3$ & $7.3 \pm 0.3$ & 9.34 & $0.024 *$ \\
\hline \multicolumn{5}{|l|}{ Signal strength } \\
\hline Macula & $70.83 \pm 10.68$ & $72.57 \pm 6.11$ & & 0.551 \\
\hline Peripapillar & $74.85 \pm 8.61$ & $73.13 \pm 7.07$ & & 0.503 \\
\hline
\end{tabular}

